\title{
Neue HKP-Leistung: Symptomkontrolle bei Palliativpatienten
}

\section{Bundesgesundheitsministerium gibt grünes Licht}

» Vertragsärzte können künftig im Rahmen der häuslichen Krankenpflege (HKP) Symptomkontrolle bei Palliativpatienten verordnen. Einer Vorgabe aus dem Hospiz- und Palliativgesetz folgend hat der Gemeinsame Bundesausschuss (G-BA) seine Regelungen für die häusliche Krankenpflege entsprechend angepasst. Das Bundesgesundheitsministerium hat diese Richtlinie nicht beanstandet, so dass die Regelungen damit nun in Kraft gesetzt sind. „Die Erweiterung der ,HäuslicheKrankenpflege-Richtlinie' des G-BA ist begrüßenswert. Die Symptomkontrolle ist bei Pflegefachpersonen richtig verortet. Aufgrund des direkten Kontakts zu den Patientinnen und Patienten kann so schnell gehandelt werden“, kommentiert Dr. Markus Mai, Präsident der rheinland-pfälzischen Pflegekammer, die Novellierung der Richtlinie.

Bei der neuen Leistung „Symptomkontrolle" geht es darum, dass die Pflegekräfte Krankheitszeichen und mögliche Begleiterscheinungen möglichst schnell erkennen. Dies erfolgt in enger
Abstimmung zwischen den Pflegenden und dem verordnenden Arzt. Eine Symptomkontrolle soll insbesondere bei Schmerzsymptomatik, Übelkeit, Erbrechen, pulmonalen oder kardialen Symptomen und Obstipation sowie bei der Kontrolle und Behandlung von exulzerierenden Wunden durchgeführt werden. Zudem gehört die Krisenintervention dazu, beispielsweise bei Krampfanfällen, Blutungen oder akuten Angstzuständen.

www.g-ba.de

\section{Schmerzpatienten: Mehr Achtsamkeit beim Überleiten}

\section{Schnittstellenmanagement bei chronischen Schmerzen}

» Bei einer Pressekonferenz anlässlich des bundesweiten „Aktionstags gegen den Schmerz" hat der Deutsche Berufsverband für Pflegeberufe (DBfK) erste Ergebnisse einer OnlineUmfrage $\mathrm{zu}$ „Schnittstellenmanagement bei chronischen Schmerzen" vorgestellt. Demnach besteht im Hinblick auf eine Versorgungskontinuität bei Schmerzpatienten dringender Handlungsbedarf.

Im März und April 2017 hatten sich mehr als 600 Pflegefachpersonen an der Umfrage beteiligt. Obwohl die Auswertung erst begonnen hat, zeichnen sich bereits jetzt erhebliche Informationsmängel bei der Überleitung bzw. Verlegung von Patienten/Bewohnen mit chronischen Schmerzen ab. Und sie sind häufig mit unangenehmen Konsequenzen für die Betroffenen verknüpft. „Was sich sehr deutlich zeigt ist ein dringender Handlungsbedarf, um die Patientensicherheit und die Versorgungskontinuität an Schnittstellen zu verbessern. 86\% der antwortenden Kolleginnen und Kollegen beschreiben die Situation als unbefriedigend“, berichtete DBfK-Präsidentin Prof. Dr. Christel Bienstein. „Es werden gerade in Bezug auf chronische Schmerzpatienten große Informationsdefizite geschildert, die häufig zu Missverständnissen, Versorgungsbrüchen oder auch Wiedereinweisungen (dem sogenannten Drehtüreffekt) füh-

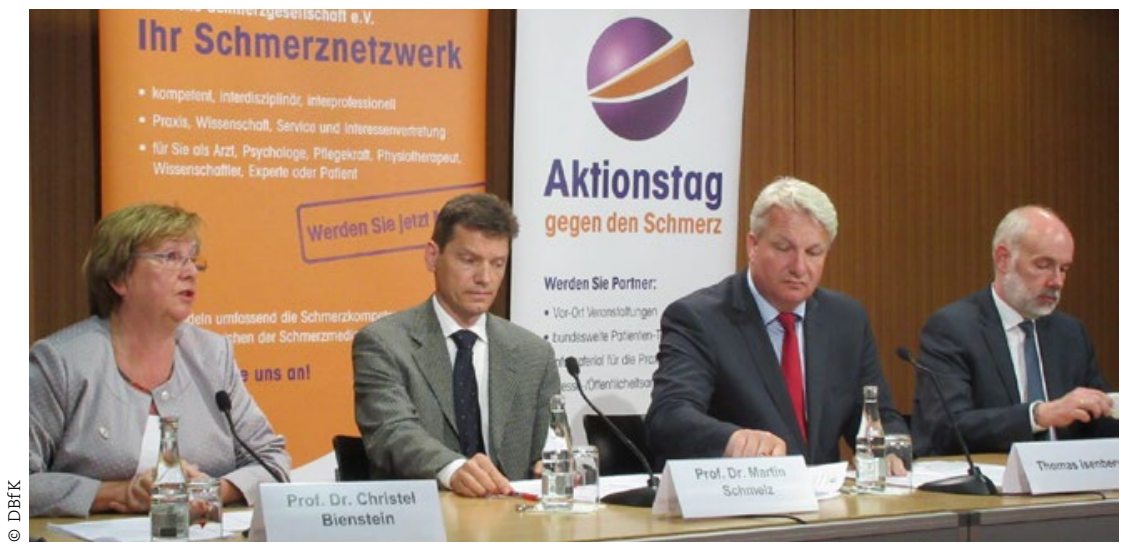

Pressekonferenz anlässlich des Aktionstages gegen den Schmerz 2017, links im Bild DBfK-Präsidentin Prof. Christel Bienstein ren. Selbst gut eingestellte Schmerzpatienten werden dadurch in ihrem Befinden oft wieder weit zurückgeworfen, müssen unnötig Leiden und Schmerzen ertragen“, sagte Bienstein weiter. „Oft sind es Unwissenheit, Gedankenlosigkeit, Zeitdruck, Pflegefachkräftemangel, schlechte Planung, lückenhafte Berichte und Dokumentationen, Fehleinschätzungen oder Inkompetenz, manchmal auch Vorurteile, die dem zugrunde liegen. Aber damit dürfen wir uns nicht abfinden."

Sie verwies darauf, dass die Erkenntnis über die Relevanz einer guten und individuellen Schmerzbehandlung sich zunehmend durchsetze. Moderne Medizin kombiniert mit fundiertem Pflegewissen könnten in den meisten Fällen Schmerzen wirksam lindern und für eine gute Lebensqualität sorgen. Gerade bei Patienten mit chronischen Schmerzen komme es dabei sehr darauf an, eine gut eingestellte Schmerzbehandlung kontinuierlich fortzuführen. Daher beschäftige sich eine Expertengruppe des DBfK mit diesem Thema und habe die Online-Umfrage initiiert. Diese werde - ausgehend von den Ergebnissen der Umfrage - weiter am Thema bleiben und Empfehlungen erarbeiten. 